\title{
A New Look at Infant Pointing
}

\author{
Michael Tomasello, Malinda Carpenter, and Ulf Liszkowski \\ Max Planck Institute for Evolutionary Anthropology
}

\begin{abstract}
The current article proposes a new theory of infant pointing involving multiple layers of intentionality and shared intentionality. In the context of this theory, evidence is presented for a rich interpretation of prelinguistic communication, that is, one that posits that when 12-month-old infants point for an adult they are in some sense trying to influence her mental states. Moreover, evidence is also presented for a deeply social view in which infant pointing is best understood - on many levels and in many ways - as depending on uniquely human skills and motivations for cooperation and shared intentionality (e.g., joint intentions and attention with others). Children's early linguistic skills are built on this already existing platform of prelinguistic communication.
\end{abstract}

Human beings communicate with one another in unique ways. Most obviously, humans communicate with one another linguistically, that is, with socially learned, intersubjectively shared symbols of a type not used by other animal species in their natural forms of communication. But humans also communicate with one another in unique ways gesturally. Many of the most important gestures humans use-for example, for greeting or leaving, for threatening or insulting, for agreeing or disagreeing - are also socially learned, intersubjectively shared, symbolic conventions that vary across cultures in much the same way as linguistic symbols (Kendon, 2004; McNeil, 1992).

An especially important gesture that has a number of unique features is human pointing. Although there may be some variations of form (e.g., in some cultures the norm is lip- or chin-pointing), the basic interpersonal function of directing someone's attention to something is very likely a human universal (Kita, 2003). Pointing is a special gesture functionally in that directing someone's attention to something does not convey a specific meaning in the manner of most conventionalized, symbolic gestures. Rather, pointing can convey an almost infinite variety of meanings by saying, in effect, "If you look over there, you'll know what I mean." To recover the intended meaning of a pointing gesture, therefore, requires some fairly serious "mindreading."

Infants begin to point to things for other persons from around 11 to 12 months of age (Carpenter,

We would like to thank the members of the Leipzig Social Cognition Group for helpful discussions of many of the ideas in this article, and all reviewers for helpful comments on a previous draft.

Correspondence concerning this article should be addressed to Michael Tomasello, Max Planck Institute for Evolutionary Anthropology, Deutscher Platz 6, D-04103 Leipzig, Germany. Electronic mail may be sent to tomas@eva.mpg.de.
Nagell, \& Tomasello, 1998; Leung \& Rheingold, 1981). The first theoretical account of infant pointing in the modern context was that of Bates, Camaioni, and Volterra (1975), who conceptualized it as a kind of social tool use, as infants begin to use physical tools at around this same age (Piaget, 1952). Using Speech Act theory, they distinguished two types of communicative act that have formed the basis for all subsequent accounts of prelinguistic communication. Infants use protoimperative points to get the adult to retrieve an object for them: They use the adult as a tool to obtain the object. They use protodeclaratives points to get the adult to attend to an external entity: They use the external entity as a tool to obtain adult attention. The other classic account is that of Bruner (1975), who focused less on the "social tool" aspect of infant pointing and more on the adult-child social interaction involved, which, on his account, grounded infants' and adults' communicative acts in an already meaningful social exchange (see Bruner, 1983, for a review). These joint attentional formats, as they were called, were seen as a kind of scaffolding within which infants shared information and attitudes with attentive and helpful adults (see also Werner \& Kaplan, 1963, on the "primordial sharing situation").

The current theoretical debates about infant pointing and prelinguistic communication center, as so many other topics in infant cognitive development, on whether the most accurate interpretation is a cognitively rich or a cognitively lean one. More specifically, the question is whether young infants are attempting in their prelinguistic communication to influence the intentional/mental states of others (cause them to "know" something) or whether, alternatively, they are simply aiming to achieve certain

(C) 2007 by the Society for Research in Child Development, Inc. All rights reserved. 0009-3920/2007/7803-0001 
behavioral effects in others (cause them to "do" something). For example, from the lean perspective, Camaioni (1993) proposes that protoimperative gestures emerge first in development and only require the infant to understand the other as a causal agentnot a mental agent-who makes things happen behaviorally, whereas protodeclarative gestures emerge later and require the infant to understand the other as a mental agent whose attention may be directed to external entities (see also Baron-Cohen, 1989; Mundy \& Sigman, 1989, with commentaries). Moore and colleagues (Moore, 1996; Moore \& D'Entremont, 2001) have taken a more thoroughgoing lean position and claimed that even protodeclarative gestures - initially, at least - are not directed at the intentional/mental states of others, but are simply directed at gaining adult attention to the self (see Shatz \& O'Reilly, 1990, for a related view).

In the current article, we follow the lead of Tomasello, Carpenter, Call, Behne, and Moll (2005; see also Tomasello, 2006) and Liszkowski (2005, 2006) in defending a rich interpretation of prelinguistic communication, that is, one that posits that when young infants point for an adult they are in some sense trying to influence her intentional/ mental states. Moreover, we also argue and present evidence for a deeply social view in which infant pointing is best understood - on many levels and in many ways - as depending on uniquely human skills and motivations for cooperation and shared intentionality, which enable such things as joint intentions and joint attention in truly collaborative interactions with others (Bratman, 1992; Searle, 1995). After a brief theoretical introduction to the nature of human pointing, we defend these theses by reviewing recent research on infant communicative pointing. We then look at recent research on other social-cognitive skills that infants would need to possess if indeed the rich interpretation of infant communication is correct, and we also provide a brief comparison of infant pointing to the "pointing" of our nearest primate relatives. We conclude with a defense of the claim that children's initial skills of linguistic communication emerge on the heels of their initial pointing gestures - and for the same basic communicative functions - because these two forms of interpersonal communication share a common social-cognitive, social-motivational infrastructure of shared intentionality.

\section{Pointing Basics}

The first thing to note is that, by itself, pointing is nothing. If you and I are walking down the street talking about the weather, and I stop and point for you in the direction of a bicycle leaning against a tree, without any other context, you will be totally mystified as to what I could possibly be intending to communicate. The reason why you will be mystified in this situation is that you do not know either what I am directing your attention to (what I am referring to) or why I am directing you to it (what is my motive).

With regard to the what question, Wittgenstein (1955) demonstrated decades ago that pointing always underdetermines the intended referent without some "form of life" or shared context within which the pointing occurs. Am I pointing at the whole bicycle? Or the special kind of polyvinyl seat? Or the color? Or the metal material it is made of? The possibilities are limitless, and demonstrate, perhaps surprisingly, that the pointing gesture can actually indicate radically different perspectives on one and the same perceptual situation. Pointing simply directs someone's attention to a location in the perceptual environment, but to correctly identify the intended referent requires that the communicator and the recipient know together that the indicated location is in some way relevant to some larger context they share (see Sperber \& Wilson, 1986, on the key role of relevance assumptions). We will call this larger context, following Clark (1996), common ground or, sometimes (when we wish to emphasize the shared perceptual context), the joint attentional frame.

And it must be emphasized that the common ground or joint attentional frame within which pointing gains its meaning is, of necessity, common or joint: We know some things or are attending to some things together. What we mean by "know together" is simply that two individuals both know that they both know; what we mean by "know" is attend or understand. (Thus, we are not attempting to address the large and complex philosophical literature on the nature of mutual knowledge nor the philosophical use of the word "know," which typically implies justified true belief.) To illustrate, suppose that you have been thinking about getting some special tires for your bicycle, but you did not discuss this with anyone, and the bicycle I am pointing to has tires of just this type. When I point to the bicycle, therefore, you will probably say to yourself something like "He's pointing to a bicycle with just the kind of tires I need, but there is no way he can know this. So he must be pointing to something else." Of course you might suppose that I divined your need in some other way (I read your secret diary), but for you to interpret my point as indicating the tires, we must both know together that I have indeed divined your 
need (Clark \& Marshall, 1981). It is important that common ground need not be personal in the sense that you and I have personally experienced things together. As Clark (1996) notes, common ground comes in many forms, including shared knowledge among members of the community even though the two communicators have never met before. Clark posits that the most direct form of common ground is perceptual copresence-we both are perceptually attending to something and both know together that we are-and this is essentially what we are calling the joint attentional frame.

In addition to knowing exactly what in their common ground a pointer is referring to, recipients must also determine why the communicator is pointing. Thus, even given that I am pointing at the bicycle as a whole, why am I doing that? Perhaps I know that you have been looking for one of that style to purchase. Or perhaps I want you to steal it for me. Or perhaps I am informing you that your ex-boyfriend is in the vicinity. Again, the possibilities are limitless. The more general question you must now ask yourself is: Why is he pointing to that bicycle for me? What does he want me to know or to do? This means that we must now distinguish at least two levels of intentionality involved in every communicative act: the communicator's more narrow referential intention of directing the recipient's attention to something, and his wider social intention or motive for directing her attention there in the first place, in the sense of what he wants her to know or to do (the communicator will have, in addition, individual goals determining why he is communicating at all).

As Grice (1975) first noted, human motivations for communicating are mainly cooperative. Thus, of Searle's (1999) five general types of speech act motives, the three most basic may be characterized in terms of helping and sharing (the other two are also cooperative in different ways):

- informative (assertive): the communicator wants the recipient to know something that he thinks she will find useful or interesting - he is helping her by informing her;

- requestive (directive): the communicator wants the recipient to do something that will help him, the communicator, in some way (including by providing needed information, as in questions); and

- expressive: the communicator wants the recipient to feel some attitude or emotion that he is already feeling - he wants her to share this attitude or emotion with him.
Thus, I may direct your attention to the bicycle in order to help you by informing you of the location of your lost bicycle (an informative motive), or I may direct your attention to the bicycle in the hopes that you will bring it to me (a requestive motive). To help you to infer my social intention, I will often produce some kind of overt (emotional) expression. Thus, if I notice an especially cool bicycle and want to share this with you, I might point to it with an excited facial expression, whereas if I am requesting that you move the bicycle, I might point to it with a stern facial expression. Also of interest here is the mutual assumption of helpfulness - both communicators trust that the other will make good faith attempts to collaborate in getting the communicator's message across (Clark, 1996; Grice, 1975) — which underlies and indeed makes possible the kind of cooperative communication characteristic of the human species.

Inferring the communicator's social intention (her motive, why she is pointing for me) also depends crucially on the common ground between communicator and recipient. For example, even in the absence of any overt emotional expression, if we both know together that you are searching for your keys, then it is likely that I am pointing to them to help you find them. On the other hand, if we both know together that $I$ am searching for my keys, then it is likely that I am pointing to them to request you to help me by fetching them for me. It is important to note that if enough is shared in the context, the overt expression of either reference or motive may be eliminated without diminishing the message. Thus, in the dentist's office the dentist may simply hold out her hand, indicating that she wants an instrument, and the assistant, based on shared knowledge of the procedure, puts the correct one in her hand without the intended referent having been indicated specifically. Conversely, the dentist may sometimes point to the instrument she wants without overtly expressing her desire per se to the assistant, as her requestive motive is mutually assumed in this mutually known context.

Crucially, as Grice (1957) first observed, cooperative communicative acts also involve in addition an intention about the communication specifically. In this analysis, when I point to a tree for you, I not only want you to notice the tree (for some reason), I also want us to notice together my desire that you notice the tree - and this additional tier is necessary to instigate in you the kinds of relevance inferences required to identify my reason for communicating in the first place (my social intention or motive). Thus, to modify an example from Sperber and Wilson (1986), suppose we are sitting on a park bench 
together, and I lean back because I am tired, which exposes a tree to your line of sight. No inferences follow. But if I lean back and point to the tree for you with an insistent expression, you must attempt to determine why I am doing this (my reason, my motive). That is, you notice that I have gone to some trouble to point out the tree to you, and to express my insistence, and this generates in you a search for some relevance within our common ground: Why does she want me to notice the tree? As I know that this is the process, I make sure that we both know together that my pointing out of the tree for you is intentional. We call this, following Sperber and Wilson, the communicative intention, and it represents my desire that we both know together that I am referring you to the tree-so that you will infer what I want you to know or to do. That this communicative intention is a natural part of human communication is evidenced by the fact that it takes a distinct effort to circumvent it. For example, borrowing again from Sperber and Wilson, if I want more wine in my glass, but think it impolite to request it directly of my host, I might simply place my empty glass in a conspicuous location so that he will see it and hopefully refill it, but without knowing that I had this in mind all along. I want the host to know about the empty glass, but not that I want him to know it. Such cases of "hidden authorship" signal an especially deep understanding of the way communicative intentions operate within the communicative act as a whole.

To summarize, Figure 1 depicts the different layers of intentionality underlying a communicator's pointing act in the current analysis. Beyond any individual goals, we must recognize: the social intention (that you do/know/feel something); the communicative intention (that we know together that I want one of these things from you); and the referential intention (that you attend to something as a way of figuring out what it is I want from you). Flipping now to the recipient's point of view, our working formula for the communicative act as a whole is: She intends that I attend to X (and wants us to know this together) for some reason relevant to our common ground. The "knowing together" part, the "common ground" part, the underlying motives for helping and sharing, and the mutual assumption of cooperation underlying all acts of human communication all rely on the basic skills and cooperative motives of shared intentionality.

\section{Infant Pointing}

In the months around the first birthday, and before they begin acquiring language in earnest, most
Individual Goals - the state of the world C wants (may be many levels).

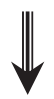

Social Intention/Motive - what $\mathrm{C}$ wants $\mathrm{R}$ to do/feel/know. Often expressed in some form of facial or other emotional expression.

- requestive: that $\mathrm{R}$ do $\mathrm{X}$, in order to help $\mathrm{C}$

- expressive declarative: that $\mathrm{R}$ feel $\mathrm{Y}$, so that $\mathrm{C}$ \& $\mathrm{R}$ share that feeling

- informative declarative: that $\mathrm{R}$ know $\mathrm{Z}$, which $\mathrm{C}$ intends as helpful

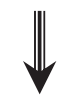

Communicative Intention - that $\mathrm{C}$ and $\mathrm{R}$ know together that $\mathrm{C}$ is attempting to communicate, so that $\mathrm{R}$ will attend to the referential act and thereby work to infer C's social intention. Often expressed in things such as ostensive eye contact.

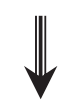

Referential Intention - that $\mathrm{R}$ attend to (identify) a specific referent. Most often expressed in gesture or language.

Figure 1. Different levels of goals/intentions underlying pointing as a human communicative act, from the communicator's perspective $(\mathrm{C}=$ communicator; $\mathrm{R}=$ recipient $)$. Arrows indicate that the higher goal/intention is carried out by means of the lower one.

infants in Western culture begin pointing, with some evidence that this is a widespread, if not universal, pattern cross-culturally (Butterworth, 2003). Our central question is the degree to which, and the ways in which, infant pointing shares all of the socialcognitive complexities of the adult version of this communicative gesture, as just elaborated.

\section{Infant Pointing in Context}

There are surprisingly few systematic studies of infants pointing in their everyday lives. Almost all such studies, including the original Bates et al. (1975) study, have been primarily concerned with children's language development, and so have viewed pointing and other gestures through this lens - to the neglect of other interesting and important aspects of the process.

Figure 2 presents some parent observations of three 11- to 14-month-old infants' pointing (selected from a larger set of observations) in the context of their everyday social interactions from the study of Carpenter et al. (in preparation). The main thing to notice is that, although the particulars differ greatly, the kinds of things that are going on seem very similar to the kinds of things going on in adult pointing, with great variety in the different messages conveyed. Thus, in the examples involving requests / 


\begin{tabular}{|c|c|}
\hline Jaron $11 ; 5$ & J points to the window when he wants it open. \\
\hline Jaron $11 ; 5$ & $\begin{array}{l}\text { J is pushing his stroller. He turns around and points behind him, } \\
\text { looks to Mom, then points ahead of him and looks to Mom. }\end{array}$ \\
\hline Jaron $11 ; 13$ & $\begin{array}{l}\text { J, alone in his room, starts crying. Mom rushes in and asks "What } \\
\text { happened?" J points to the coat rack which had fallen down. }\end{array}$ \\
\hline Jaron $11 ; 18$ & As Dad prepares to leave, J points to the door. \\
\hline Jaron $11 ; 19$ & $\begin{array}{l}\text { Mom pours water into J's glass. A few minutes later, J points to the } \\
\text { pitcher to tell her to pour him some more. }\end{array}$ \\
\hline Jaron $12 ; 0$ & $\begin{array}{l}\text { Mom tells J not to touch her hot teacup. Later he points to it and } \\
\text { says "No", looking to her for confirmation. }\end{array}$ \\
\hline Jaron 12;2 & $\begin{array}{l}\text { When Mom asks where J got something from he points under the } \\
\text { table and says "There." }\end{array}$ \\
\hline Jaron 12;26 & $\begin{array}{l}\text { J points to Grandpa's chair and says "There" to ask him to sit down } \\
\text { next to him. }\end{array}$ \\
\hline Jaron 13;3 & $\begin{array}{l}\text { J watches quietly as Dad arranges the Christmas tree. When } \\
\text { Grandpa enters the room J points to the tree and says "Oh!" }\end{array}$ \\
\hline Jaron $13 ; 17$ & $\begin{array}{l}\text { When J bumps into something or falls, he points to the offending } \\
\text { piece of furniture or location on the floor. }\end{array}$ \\
\hline Alex $12 ; 10$ & $\begin{array}{l}\text { When A hears an airplane from in the house, he points through the } \\
\text { window to the sky (the airplane is not visible). }\end{array}$ \\
\hline Lisa $13 ; 15$ & $\begin{array}{l}\text { After eating L points to the bathroom, anticipating going to wash } \\
\text { hands. }\end{array}$ \\
\hline Lisa 13;20 & $\begin{array}{l}\text { Mom is looking for the missing refrigerator magnets. L points to } \\
\text { the basket of fruit where they are (hidden under fruit). }\end{array}$ \\
\hline Lisa $13 ; 23$ & $\begin{array}{l}\text { L pulled the heating lamp halfway off the wall. Mom calls Dad. } \\
\text { Dad comes in and L points to the lamp to show what happened. }\end{array}$ \\
\hline Lisa $13 ; 26$ & $\begin{array}{l}\text { L points to the door and says "Papa" when it is about time for him } \\
\text { to come home. }\end{array}$ \\
\hline Lisa $14 ; 13$ & $\begin{array}{l}\text { Mom holds down L's plate so L cannot bang it anymore. L looks to } \\
\text { her aunt sitting nearby and points to Mom's hand, to request help } \\
\text { removing Mom's hand. }\end{array}$ \\
\hline Lisa $14 ; 13$ & $\begin{array}{l}\text { Mom is bringing L's highchair to the table; L points to where it } \\
\text { goes. }\end{array}$ \\
\hline Lisa $14 ; 14$ & $\begin{array}{l}\text { Dad puts tights on L's head like a hat several times; Dad stops. L } \\
\text { gives the tights to her aunt, who puts the tights aside. L protests } \\
\text { and points to herself, asking to continue the game. }\end{array}$ \\
\hline Lisa $14 ; 17$ & When L wants to get into her stroller or highchair she points to it. \\
\hline
\end{tabular}

Figure 2. Some parent observations of three 11- to 14-month-old infants' pointing in the context of their everyday social interactions-from the study of Carpenter et al. (in preparation). 
imperatives, these infants pointed not only to objects they wanted to have but also to the object involved when, for example, they wanted the window opened or the glass filled with water-thus requesting not an object but an action. They also pointed to a desired location, for example, when they wanted a chair or their own bodies moved to and placed in a certain place. These are all clearly requests, but only some of them are concerned with obtaining objects as in classical protoimperatives. And the pointing itself is sometimes to the object (the action to be performed being assumed) but sometimes to a location (the object and action being assumed), suggesting different joint attentional frames in the different cases.

In addition, these infants also pointed to express a wide array of other messages. For example, they pointed to the door through which Papa was preparing to leave, to the object they were forbidden to touch, to the location where an object was previously found, to a sight new and interesting for Grandpa (not the infant), to an object that had previously hurt them, and to the location where an exciting event occurred just previously. These could all be classified as protodeclaratives, in the sense that the infant's intention is to direct or share attention to something, but in these different observations the infants are directing and sharing attention to very different aspects of the target events, and they are doing so for a wide variety of different reasons, including both anticipating and remembering nonpresent events - again, significantly different from classical protodeclaratives used only for obtaining adult attention to objects.

Nevertheless, despite the superficial similarity to observations of adult pointing - in the sense of a variety of contexts, motives, and messages (although obviously in somewhat simpler situations) - we still cannot tell from natural observations alone the nature of the social-cognitive processes involved in these interesting communicative acts. We must supplement these observations with experiments investigating the social-cognitive and social-motivational processes involved, either directly in studies of pointing or indirectly in studies of related developmental phenomena. We thus look now at experimental evidence for each of the major components of the pointing act, as outlined above, in the earliest pointing gestures of infants from around the first birthday.

\section{The Joint Attentional Frame or Common Ground}

Human infants follow adult pointing gestures to distal targets, and check back with the adult to make sure of her target, from around the first birthday (Carpenter et al., 1998). Soon after that, they know what such gestures mean in the sense that they do not just follow the point to a location and then attend to whatever grabs their attention, but rather they seek the relevance of the point to some joint attentional frame, or common ground, they share with the pointer. For example, in the preestablished context of a hiding-finding game, Behne, Carpenter, and Tomasello (2005) hid a toy in one of two buckets and then, subsequently, pointed to the toy's hiding place to help the child to find it. Infants from 14 months of age successfully inferred the hidden toy's location, presumably based on the common ground within which both the infant and adult knew together that the infant was seeking this toy (and the adult wanted to help). Although this task seems completely trivial to human adults, it is not. Great apes typically follow the pointing gesture to the correct referent, the bucket, but they do not then know what the human intends by his pointing gesture and so fail to find the hidden food (see the Phylogeny section for a fuller treatment of apes in this task).

The critical role of common ground and/or joint attention in infants' comprehension of communicative acts is illustrated in two sets of experiments. First, a bit indirectly, Tomasello and Haberl (2003) had 12-month-old infants engage with an adult in joint attentional interactions with each of two objects, and then the adult left the room-while the infant played with a third object with an assistant. When the adult returned and gestured ambiguously in the direction of all three objects, grouped together, and excitedly said to the infant "Wow! Cool! Can you give me that?," infants gave her the one that they had not shared with her in joint attention previously - thus illustrating their differentiation of objects previously shared and previously not shared with that adult. And Moll and Tomasello (in press) found that just observing while the adult inspected the first two objects on her own was not sufficient for 14-monthold infants to know that she had experienced them; the sharing of attention to the objects was indeed critical. These studies thus demonstrate that infants as young as 12-14 months of age identify the referent of an ambiguous request in terms of some shared experiences they have previously had with that adult.

More directly, in a second set of studies Liebal, Behne, Carpenter, and Tomasello (2007) focused in particular on infants' comprehension of pointing, and on how infants identify not the referent but rather the motive behind a pointing gesture. In this study, 18-month-old infants and an adult cleaned up together by picking up toys and putting them in a basket. At one point the adult stopped and pointed to a target toy, which infants then picked up 
and placed in the basket. However, when the adult pointed to this same toy in this same way but in a different context, infants reacted differently: When the infant and adult were engaged in stacking rings on a post, children ignored the basket and brought the target toy back to stack it on the post. The crucial point is that in both conditions the adult pointed to the same toy in the same way, but the infant extracted a different meaning in the two cases - based on the two different joint attentional frames involved. And the jointness is again a crucial component here. Thus, in a control condition, the infant and adult cleaned up exactly as in the shared clean-up condition, but then a second adult who had not shared this context entered the room and pointed toward the target toy in the same way as the first adult in the first condition. In this case infants did not put the toy away into the basket, presumably because the second adult had not shared the cleaning-up context with them. Rather, because they had no shared frame with this adult, they seemed most often to interpret the new adult's point as a simple invitation to notice and share attention to the toy. Comparison of these different experimental conditions shows quite clearly that infants' interpretation of an adult pointing gesture depends on their recently shared experience (joint attention, common ground) with that specific adult. In a second study this was also confirmed by having the two adults each play a different game with infants, in which case they interpreted each adult's pointing gestures as relevant to the particular game they had shared with that particular adult previously.

The basic theoretical point is this. If the successful interpretation of a pointing gesture depends, as argued in the previous section, on communicator and recipient making contact with some common ground or joint attentional frame, there is good evidence that infants in the period from 12 to 18 months of age do indeed construct with others in their activities with objects the kinds of joint attentional frames necessary for human-style cooperative communication (see, e.g., Bakeman \& Adamson, 1984). This then enables them to interpret potentially ambiguous communicative acts, including ambiguous pointing gestures, both in terms of what referent the communicator is indicating and what motive she has for indicating it.

\section{Reference}

Infants follow the gaze of others to targets from very early ( $D^{\prime}$ Entremont, Hains, \& Muir, 1997), but the infant following adult gaze (or even pointing) to a target does not constitute a successful act of reference. A successful act of reference occurs when one individual intends for another to attend to something within some larger communicative context, and the other recognizes this intention and complies with it.

Evidence that infants comprehend an act of reference as an intentional direction to focus on a referent comes, again, from the study of Behne et al. (2005). Recall that when the adult pointed to a bucket for 14-month-old infants in the context of a hidingfinding game, infants followed her point there, and, importantly, also made the inference that she intended for them to attend to the bucket as location, as this was what was relevant to their joint activity in the hiding-finding game. Crucially for current purposes, in a control condition, Behne et al. (2005) had the adult hold her hand in a pointing shape directed at the correct bucket (just as before), but while distractedly inspecting her wrist - so that even though her actions resembled referential pointing, it was clear from her overall demeanor that she was engrossed in a private activity, not doing something such as pointing for the infant. In this case, infants did not find the hidden toy, presumably because they did not see the adult's behavior (including her protruding finger) as an intentional act of attention directing - referring-within the context of some larger joint attentional frame or common ground. (Note that the same pattern of results was found for communicative versus distracted gazing at the correct bucket, showing that children did not ignore the adult's point in the control condition above simply because she was not looking at the bucket.)

When infants produce points, there is also very good evidence that they themselves intend for the other to attend to a referent. This is not a foregone conclusion. Indeed, Moore and colleagues have expressed skepticism that 12-month-olds produce gestures as an attempt to direct the attention of others to external entities. Thus, Moore and Corkum (1994) contend that early (declarative) pointing is mostly aimed at gaining adults' positive emotion to the self, and Moore and D'Entremont (2001) claim that it is the adult's reaction to the infant, instead of to the external entity, that serves as a reinforcer for the pointing behavior. The main evidence for this skeptical interpretation is that infants sometimes point to things for the adult that she, the adult, is already looking at, and so the pointing cannot be an attempt to direct her attention to something new, as they are already both attending to the object. Moore and $\mathrm{D}^{\prime}$ Entremont thus argue that what the infant is really doing is not referring to the object at all, but only attempting to get an emotional reaction from the adult to the self. 
Liszkowski, Carpenter, Henning, Striano, and Tomasello (2004) directly tested the hypothesis of Moore and colleagues, along with some others, in an attempt to determine whether infants' declarative points are attempts to direct adult attention to a referent - so that they can then share their attitude about it. Pointing was elicited from infants in a situation in which a declarative motive would be likely, with objects such as puppets either suddenly appearing or engaging in interesting actions from afar. The adult's reaction was experimentally manipulated and the infant's response to this reaction was observed. The main finding with respect to reference (more on this study with respect to motivation below) was that when the adult responded to the infant's point by simply emoting directly to her, ignoring the referent, infants showed signs of dissatisfaction by repeating their pointing, in an attempt at message repair, and they pointed less often over trials - again indicating dissatisfaction with the adult's response that ignored the intended referent. Even more directly, using the same basic methodology, Liszkowski, Carpenter, and Tomasello (2007b) had the adult either correctly identify the infant's intended referent or else misidentify it by alighting on a different nearby object (in both cases with positively expressed emotion and gaze alternation). When the adult correctly identified the intended referent, infants simply continued sharing attention and interest with him, but when the adult alighted on the incorrect referent, infants repeated their pointing to the intended referent in an attempt to direct him there.

Interestingly and importantly, 12-month-old infants can also make reference in their pointing to absent entities. This is apparent in a number of the observations of Figure 2, as 11- through 13-month-old infants refer to events that happened in the near past or will happen in the near future. More systematically, Liszkowski, Carpenter, and Tomasello (2007a) exposed infants to targets likely to elicit declarative pointing, and then, after a while, the targets disappeared. The majority of infants - both those who had pointed to the visible target and those who had not-pointed for an adult to the location where the visible target used to be, especially if the adult had not seen the target previously. Pointing to absent referents is important because it makes it clear that pointing infants are not doing very low level, behavioristic things like attempting to get the other person to orient bodily to perceptible entities, but rather they are attempting to get the other person to orient mentally to some nonperceptible entity that they have in mind (see also Saylor, 2004).
The overall point is this. In both their comprehension and production of pointing gestures, infants by 12-14 months of age demonstrate an understanding of acts of reference as intentional acts intended to induce the other to attend to some particular external entity, even an absent entity, as a part of some larger communicative act occurring within some joint attentional common ground. This process involves much more than simply gaze following or point following or gaining attention to the self. It involves a communicator's intention to direct a recipient's attention to a particular referent so that the recipient, by identifying this intended referent via some relation to their common ground, will make the needed relevance inferences and so comprehend her, the communicator's, overall social intention.

\section{Social Intention (Motives)}

Pointing to communicate thus always involves as one component the pointer inviting the recipient to attend to some referent. This is the main function of the indicating finger (Brinck, 2004). But, as emphasized above, the pointer is always inviting the recipient to attend to some referent for a reason. The pointer has some larger social intention; she wants to influence or change the recipient in some way. Her particular motive - exactly what she wants the recipient to do or how she wants him to be-is sometimes (though not always) expressed via some overt expression of the pointer's emotional state.

As noted at the outset, infant pointing has classically been hypothesized as emanating from two motives: declarative and imperative. We think the situation is actually a bit more complex than this. In particular, we think the declarative motive has two important subtypes, and that the imperative motive actually involves a continuum from something like ordering (forcing) to something like suggesting (influencing choice). In addition, in order to integrate communication into the infant's other cognitive activities, we think it is best to think of all the different motives more broadly in terms of shared intentionality, specifically, infants' skills and motivations for cooperatively helping and sharing with others.

In the original Bates et al. (1975) formulation, protodeclarative pointing was analogous to a declarative sentence, such as "The cat is on the mat". Statements of this type have truth-values that indicate how well they fit to the true state of the world, what Searle (1995) calls a mind-to-world direction of fit. However, in many subsequent analyses, the prototype of declarative pointing is when the infant points to, for example, an interesting animal in the 
distance, expresses emotions, and alternates gaze to the adult. The infant is interested or excited about the new animal, and seemingly wants to share her excitement with the adult by getting him to look at it along with her and share a reaction (hopefully the same) to it. This is not much like a declarative statement with a truth-value, as its motive seems to be very different. We thus believe that we should distinguish between (i) declaratives as expressives, in which the infant seeks to share an attitude with an adult about a common referent, and (ii) declaratives as informatives, in which the infant seeks to provide the adult with needed or desirable information (which he currently does not have) about some referent. Experimental research has established each of these as an independent motive for infants at around their first birthdays.

First, as noted above, Liszkowski et al. (2004) elicited pointing from 12-month-olds in a situation in which a declarative motive - of the expressive subtype - would be likely (e.g., novel and interesting objects suddenly appearing at some distance), and then experimentally manipulated the adult's reaction. Specifically, the adult reacted to the infant's pointing by

- emoting positively toward the infant without looking at the event - on the hypothesis that the infant wants adult attention and emotion to the self, à la Moore and colleagues, not attention to the referent (Face condition);

- looking to the event without looking to the infant-on the hypothesis that the infant simply wants to direct the adult's attention to the event, not share attention and interest (Event condition);

- doing nothing - on the hypothesis that the infant is pointing for the self only, or is not attempting to communicate at all (Ignore condition); and

- alternating gaze between the infant and the event while emoting positively - on the hypothesis that the infant wants to direct adult attention to the referent, so that they can share attention and interest in the event together (Joint Attention condition).

Infants' reactions to these reactions were then noted in an attempt to establish the infants' motive for pointing. Results showed that when the adult simply expressed positive emotions to the infant while ignoring the indicated referent (Face condition), or when the adult simply looked to the indicated referent while ignoring the child (Event condition), infants were not satisfied. In comparison with the Joint Attention condition, in which infants typically gave one long point, infants in these conditions (as well as in the Ignore condition) tended to repeat their pointing gesture more often within trials - apparently as persistent attempts to establish shared attention and interest. Moreover, infants in these conditions (as well as in the Ignore condition) pointed less often across trials than in the Joint Attention condition - apparently indicating growing dissatisfaction with this adult as a communicative partner as she did not respond by sharing infants' attitude to the referent. Even more directly, using the same basic design, Liszkowski et al. (2007b) had the adult correctly identify the infant's intended referent, but in different conditions the adult either (i) expressed interest ("Cool!") or (ii) expressed disinterest ("Uh ...") in this referent. When the adult expressed disinterest, infants did not prolong or repeat their pointing within trials, presumably because they understood that the adult did not share their enthusiasm, and they also decreased pointing for this adult across repeated trials compared with when the adult expressed interest. These results specifically isolate the infants' motive to share their attitude with an adult in the expressive subtype of declarative pointing, their motive being that the adult not just attend to a referent but also align with their attitude about it.

Second, the informative subtype of declarative pointing occurs when the infant's intention is to help the adult (dispassionately) by providing her with information she needs or would be interested in. This motive for pointing is actually much closer to that behind most declarative statements expressed in language. To have this motive infants must have, first, an understanding that others can be knowledgeable or ignorant, and second, an altruistic motive to help others by supplying them with the needed or desirable information. In order to test whether 12-month-old infants point with such a motive, Liszkowski, Carpenter, Striano, and Tomasello (2006) placed infants in various situations in which they observed an adult misplace an object or lose track of it in some way, and then start searching. In these situations infants pointed to the needed object (more often than to distractor objects that were misplaced in the same way but were not needed by the adult), and in doing this they showed no signs of wanting the object for themselves (no whining, reaching, etc.) or of wanting to share emotions/attitudes about it. In a follow-up study, Liszkowski, Carpenter, and Tomasello (2007c) presented infants once again with an adult searching, but in this case the two candidate objects 
differed only in whether the experimenter had seen them in their current location or not (one of them he had placed aside, whereas the other had fallen away unnoticed). In this case again infants pointed more often to the object the adult had not seen in its current location (and they showed no signs of wanting the object or wanting to share emotions about it). These results suggest that when pointing declaratively infants sometimes want to do something other than share their excitement about a referent with an adult, as occurs in the classic cases; they sometimes simply want to help the adult by providing needed or desirable information for her-and these two motives are distinct.

Turning now to imperative pointing, some researchers have argued that imperatives expressed through pointing are at least potentially quite simple, based on an understanding of others as causal (not intentional or mental) agents who make things happen (e.g., Camaioni, 1993). This view is based at least partially on the fact that children with autism point imperatively but not declaratively, as do some apes when interacting with humans (Leavens \& Hopkins, 1998; Tomasello \& Camaioni, 1997). But imperatives actually form a continuum. Some are based on individualistic motives and understanding by inducing or even forcing the other, as a causal agent, to do what one wants; for example, a young infant might point to a toy with the goal that the adult retrieve it for her, with the adult understood as a kind of social-causal tool (similar to the original Bates et al., 1975 formulation). Other imperatives are based more on cooperation by telling the other what I want, as in so-called indirect requests, and hoping that she, as an intentional/ mental agent, will decide to help.

Obviously, human infants sometimes produce more individualistic imperatives to get adults to do things for them as social tools. But they also sometimes produce more cooperative imperatives in which they attempt to go through the intentional/mental states of the recipient-her understanding and motivations - in a way that more individualistic imperatives do not. It is not totally clear what kind of evidence would be persuasive that infants are sometimes using such cooperative imperatives. One indirect piece of evidence is that from a very young age infants point in other ways that are clearly cooperative and that clearly go through the intentional/mental states of the other, that is, they use both expressive and informative declaratives (as demonstrated above) as early as they use imperatives (Carpenter et al., 1998). More directly-although the evidence is only for somewhat older children at 30 months of age-when young children request something from an adult, and the adult misunderstands them but then, by luck, they get what they want anyway, they still attempt to correct the misunderstanding (Shwe \& Markman, 1997). This suggests that fairly early in development children understand that their request works not by forcing the adult into a specific action, but rather by informing the adult of their desire and then her comprehending this and agreeing to cooperate with it. We do not know precisely when this understanding first occurs in infant development.

Our contention is thus that recent research on infant pointing establishes three general classes of social intention or motive (each of which encompasses many particulars, such as those in Figure 2) that correspond to those outlined above for adults:

- they want others to feel things: expressive declaratives for sharing emotions and attitudes about things;

- they want others to know things: informative declaratives for helping others by providing them with needed or desirable information; and

- they want others to do things: imperatives or requestives (either more individualistic or more cooperative) for asking others to help them in attaining goals.

Importantly, these social intentions all involve in some way or another cooperative motives for helping and/or sharing - the two main types of motivation in shared intentionality.

\section{Communicative Intention}

One final issue concerns infants' understanding of the communicative intention. That is, the question is whether children comprehend and produce their early pointing gestures with the full adult-like schema: she intends that I attend to $X$ (and wants us to know this together) for some reason relevant to our common ground. It is not clear whether 1-year-old infants operate with the "and wants us to know this together" part of the schema, either in comprehension or production. The phenomena that require us to posit the full-fledged schema for older children and adults include, most conspicuously, hidden authorship and concealment - such acts as placing one's empty wine glass for the host to see (and fill), but not revealing that this is what one has done. Adults engage in this kind of hidden authorship quite often in cases involving politeness or other forms of concealment, whereas 1- and 2-year-olds seemingly do not engage in this behavior at all. 
However, it is possible that infants operate with some kind of primordial, undifferentiated communicative intention that contains the basic structure, but not all of the adult details. There are several lines of evidence for this. First, from around the first birthday infants clearly produce communicative acts "for" another person (or understand such acts as "for" them), as they make sure they have the attention of the other, direct the act to them, make eye contact, and so forth (see Csibra, 2003, on infants' understanding of the communicative/pedagogical intentions of others). Second, in the experiment of Behne et al. (2005), when the adult in the control condition directed her extended index finger to one of the buckets as she distractedly examined her wrist, 14-month-old infants did not see this as a communicative act "for" them, and so they did not make the appropriate relevance inference (i.e., they did not see it as informing them of the location of the hidden toy, as in the experimental condition in which the adult pointed to the bucket "for" them). Third, in the studies of Moore and D'Entremont (2001) and Liszkowski et al. (2007a) infants sometimes pointed even though the adult was already attending to the target referent, a behavior that could be interpreted as infants wanting the adult not only to attend to the object but also to know that they wanted to communicate with him about this object. Finally, in the study of Shwe and Markman (1997) children at 30 months of age corrected adult misunderstandings, even when they (by accident) got the object they wanted, suggesting that they had both the goal of getting the object and the goal of communicating successfully with the adult.

Our view is thus that infants in the second year of life comprehend a primordial version of communicative intentions in the sense that they understand when a communicator intends an act "for" someone else's benefit, and intends that both she and the recipient know this together. Coming to understand the full intentional structure of adult-like communicative intentions involving hidden authorship, concealment, and the like-presumably at around 3 or 4 years of age - is a process of differentiating the more specific means by which an initially undifferentiated communicative intention may be expressed and understood.

\section{Summary}

The debate was first framed by Shatz (1983). She claimed that children's earliest communication, including linguistic communication, is aimed only at achieving behavioral results. Young children are trying to get adults to do things, and they do not understand how the communicative process works in achieving these results. Golinkoff (1993) reviewed evidence for a contrary view, but at that time there was not so much good experimental data.

Despite the attempts of Moore and colleagues (Moore, 1996; Moore \& D'Entremont, 2001) to resurrect a variation of Shatz's proposal, we believe that the relatively new data we have reviewed here argue strongly that infants do indeed understand the most important aspects of how human-style, cooperative communication works. First, from at least their first birthdays infants are able to coconstruct with others the kinds of joint attentional frames (common ground) necessary for comprehending and producing cooperative communicative acts involving relevance inferences about communicator intentions. Second, from their earliest communicative points infants' referential intention when pointing is aimed at directing others' attention to some entity within this joint attentional frame, including sometimes absent referents, again suggesting a process of influencing other minds as the referent must in these cases be imagined. Third, infants understand and operate from the beginning with the fundamentally cooperative social motives embodied in expressives (sharing attitudes), informatives (offering help by informing), and possibly cooperative imperatives (requesting voluntary help) - motives that seem to be absent from the communication of other primates, even when they are communicating with humans. And finally, pointing infants understand from very early, albeit in a rudimentary fashion, that one achieves one's social intention mainly by making others aware of it (i.e., they understand at least something about the communicative intention), clearly an understanding of the mental states of others.

\section{Role of Shared Intentionality}

Infants thus comprehend and produce their pointing gestures - basically from their first points at around 12 months of age - in surprisingly adult-like ways, both in the sense that they are operating on a mental level and also in the sense that they are cooperating with others in acts of shared intentionality as they do so. The question now is where do these communicative skills come from ontogenetically and phylogenetically. In addressing these questions, we find even further support for the hypothesis that infant pointing depends crucially on skills and motivations for shared intentionality. 


\section{Ontogeny}

A basic question in all developmental analyses is why some competence emerges when it does in ontogeny. Answering this question often provides important information about the underlying cognitive and motivational skills involved. In the case of pointing, the specific behavioral form-distinctive hand shape with extended index finger-actually emerges reliably in infants as young as 3 months of age (Hannan \& Fogel, 1987). However, as far as anyone can tell, infants at this age are not using this hand shape for any communicative function. This is despite the fact that they also seem to have some of the needs that precipitate truly communicative pointing later in development, for example, the need for adults to do things for them, including fetching out-of-reach objects (underlying requests), and the need for adults to share emotions with them in protoconversations (underlying expressives) (see Masataka, 2003). So why do infants not learn to use the extended index finger for these social functions at 3-6 months of age, but only at 12 months of age?

Our basic answer is that 3- to 6-month-old infants do not point for others communicatively because communicative pointing requires at least some implicit understanding of the formula she intends that I attend to $X$ (and wants us to know this together) for some reason relevant to our common ground. Infants do not yet have the requisite understanding of intentions, attention, and shared attention and knowledge - nor the requisite motivations for cooperation and helping. As soon as they acquire these competencies and motivations infants begin pointing for others communicatively, suggesting some connection. Based on the best available evidence, here is when some key prerequisite social-cognitive skills emerge:

- Infants understand goals by 9 months of age (e.g., Behne, Carpenter, Call, \& Tomasello, 2005; Gergely, Nádasdy, Csibra, \& Bíró, 1995). Moreover, by 12 months of age infants know that actors actively choose means for pursuing goals, thus forming intentions, and they are even able to discern some of the reasons why an actor chooses one particular means over another (Carpenter, Call, \& Tomasello, 2005; Gergely, Bekkering, \& Király, 2002; Schwier, van Maanen, Carpenter, \& Tomasello, 2006).

- Infants understand perception by around 12 months of age (Moll \& Tomasello, 2004), and by 12 months of age they understand that actors choose to intentionally attend, for some reason, to some subset of the things they perceive (e.g., Moll, Koring, Carpenter, \&
Tomasello, 2006; Tomasello \& Haberl, 2003).

- Infants by 12-15 months of age can determine what others "know" (Onishi \& Baillargeon, 2005; Tomasello \& Haberl, 2003)

- Infants by 12-14 months of age know what they have and have not mutually experienced with another person in episodes of joint attention: what they know together (Moll \& Tomasello, in press; Tomasello \& Haberl, 2003).

- Infants by 9-12 months of age begin to understand instrumental helping toward goals (Kuhlmeier, Wynn, \& Bloom, 2003), and they actually help others instrumentally by $12-14$ months of age (Liszkowski et al., 2006; Warneken \& Tomasello, 2006, in press).

- Infants by 14 months of age form joint goals with others in cooperative activities (Warneken \& Tomasello, in press).

Together, then, the studies in these different areas suggest that infants by around 12 months of age for the first time understand (i) something about the choices people make in action and perception (their intentions and attention); (ii) something about why people make these choices, in terms of some higher guiding goal and also possible constraints; (iii) what knowledge they do and do not share with others based on what they have experienced together with them in joint attentional interactions; and (iv) the basic cooperative motives. It would thus seem that infants only begin to possess the basic social-cognitive and social-motivational skills for engaging in human-style cooperative communication at around 12-14 months of age.

Our overall argument is that the skills that really make a difference here are those of shared intentionality (although of course skills of individual intentionality are necessary as well). The problem is that in the ontogenetic theory of Tomasello et al. (2005), there are two lines of development that converge to result in skills of shared intentionality. Specifically, shared intentionality emerges when the sharing line of development-present from soon after birth as infants share emotions with others in protoconversations - interacts with the intentionreading line of development that consolidates at around 9-12 months of age. It is thus not possible by looking at the naturally occurring developmental pathway to specify whether the emergence of infants' communicative pointing at around 1 year of age is due to emerging skills of individual or shared intentionality, as both emerge together. But there is another tack we can take on this question, and this is to look at the case of our nearest primate relatives, 
who do not point for one another but who do sometimes point for humans, to see whether this sheds some light on the matter.

\section{Phylogeny}

In their natural communication, humans' closest primate relatives do not point for one another (nor do any other animal species - but see Vea \& Sabater$\mathrm{Pi}, 1998$, for one possible incident). However, chimpanzees and other apes growing up in human captivity do learn to "point" to out-of-reach food so that a human will retrieve it for them (Leavens \& Hopkins, 1998), and sometimes for other things they desire (e.g., locations to which they wish access). Approximately $60-70 \%$ of all captive chimpanzees engage in this behavior when presented with the appropriate situation, spontaneously with no training from humans. Typically, they are doing this through caging, and so they orient their body toward the out-of-reach food, and thrust their fingers and hands in the caging toward the food as well. They are not reaching directly for the food, because when a human is not present they do not engage in this behavior.

Leavens, Russell, and Hopkins (2005) have documented that this "pointing" behavior is used quite flexibly. For example, if several different types of food are available, chimpanzees will point to the most desirable one, and they will continue pointing to the most desirable one even if given a less desirable one. They thus point to a specific object, and they do so persistently until they get what they want. Two other interesting variations are as follows: First, when some human-raised apes observe a human hiding food in an open area outside their cage, when another, naive human comes by many hours later, they will point for him to the location where the food is hidden (Menzel, 1999). Second, when apes observe that a human needs a tool to retrieve food for them, and then that tool is hidden when the human is away, when the human returns they will point to the location of the hidden tool that he needs if he is to get them food (Call \& Tomasello, 1994). Even if this is a request that the human retrieve the tool (so that he can retrieve the food), its indirectness is still remarkable.

Importantly, apes do not produce, either for humans or for other apes, points that serve functions other than the imperative/requestive function. That is, they do not point declaratively to simply share interest and attention in something with another individual, and they do not point informatively to inform others of things they want or need to know. It is thus possible that ape pointing for humans relies on somewhat different social-cognitive and social-motivational skills than does human pointing. One possible indication of this is that apes are not very skillful at comprehending informative pointing gestures.

If a human points and looks toward some food that an ape currently does not see, and by following the point/look the ape comes to see the food, he will go get it. In this sense, one could say that the ape understood the human's point as an attention-director. But a seemingly minor change in this procedure leads to drastically different ape behavior-which might lead us to reassess the simpler situation. In object choice experiments - in which a human attempts to help the ape locate hidden food by pointing to its location (similar to that of Behne et al., 2005, with infants, described above) - apes generally perform quite poorly. They can be trained to do the task, and some individuals - especially those with much human experience - seem to do a bit better than others. But, in general, very few apes understand the pointing gesture in this context spontaneously (see Call \& Tomasello, 2005; Miklosi \& Soporoni, 2006, for reviews). (It is interesting that some domesticated animal species (especially domestic dogs) and human-trained animals (e.g., human-trained dolphins) are reasonably skillful in this object choice task (see Hare \& Tomasello, 2005, for a review). The explanation for this at the moment is not clear, with one possibility being that they understand the pointing not as informative but rather as a command to go to a location.

Task failures may be explained in an almost infinite number of ways. But the results of a follow-up study constrain the possibilities considerably. Hare and Tomasello (2004) conducted a competitive version of the object choice task in which, instead of pointing, a human reached toward the correct bucket, but due to the physical constraints in the situation, was unable to grasp it. Now the chimpanzees knew where the food was-whereas the exact same individuals did not know where it was in a standard informing (pointing) version of the task. They were seemingly able to infer: She wants to get into that bucket for herself; therefore, there must be something good in there. But they were not able to infer: She wants me to attend to that bucket for some reason relevant to our common ground. Thus, even though the superficial behavior of the human was highly similar in the two versions of this task - arm extended toward correct bucket-apes' understanding of the human's behavior was very different, presumably because she was expressing an individual intention not a communicative intention. 
What are we to make of the apes' behavior in these studies? One plausible interpretation is as follows: Human infants find the object choice task trivially easy because (i) they have created with the adult a shared goal of searching for the hidden object, which creates a joint attentional frame of things relevant to that shared goal (a shared space of possible referents and messages); and (ii) child and adult mutually assume that the adult is trying to be cooperative. In contrast, apes typically do not know what the human is attempting to communicate in this situation because, on our account: (i) they have not created with the human any kind of joint goal or joint attentional frame of things relevant to a shared activity; and (ii) they do not share with the human the mutual assumption of partner cooperativeness. This means that the apes do not, indeed cannot, make the appropriate relevance inferences because they are missing the basic premise that the communicator is attempting to inform them cooperatively of something in their common ground that she thinks they will find relevant to their own concerns - even though they can make other inferences about what a human competitor is doing in a goal-directed act. And so apes simply do not understand why the human is pointing to the bucket, even if the communicative intention is overtly expressed; the gesture is an inexplicable, irrelevant act. The ape is searching for the food individually and so she follows the human's point to the bucket, but then she says to herself, as it were: "A bucket. So what? Now where's the food?" The bucket is not relevant for her, and it has not occurred to her that the human might be pointing altruistically to inform her of things he thinks she will find relevant.

In general, although we do not have as much solid data as we would like for some issues, the overall common factor among these critical differences in ape and human communication would seem to be something in the direction of shared intentionality. Indeed, there is a growing body of evidence that whereas apes do understand the basics of intentional action - they understand others' goals and perceptions, for example - they do not participate in shared intentionality (see Tomasello et al., 2005, for a review). Apes' understanding of others' goals and perceptions supports both the production and comprehension of some forms of imperative pointing, which presupposes an understanding of intentional agents who make things happen. But apes are not motivated to simply share information and attitudes with others, nor do they comprehend when others attempt to communicate with these motives. Apes also do not share common conceptual ground or joint attentional frames with others - that is, contexts that are "known together" - nor is their communication premised on mutual assumptions of cooperativeness among partners. This means that apes' communication, both with one another and with humans, is more individualistic, whereas humans' communication is more cooperative in the sense that it is all about helping and sharing within the context of various kinds of common conceptual ground with other persons. Without such shared intentionality, human-like cooperative communication is simply not possible.

\section{Into Language}

Also relevant for characterizing the nature of infant pointing is its relation to the other forms of communication that follow it ontogenetically, specifically linguistic conventions. Our claim here is that children's initial skills with a conventional language emerge on the heels of their initial skills with pointing - typically by only a couple of months - because both of these forms of infant communication are learned and used within the same interpersonal nexus of shared intentionality - with pointing paving the way. Indeed, the critical role of shared intentionality in language learning and use is the central premise of the social-pragmatic theory of language acquisition, as espoused by Bruner (1983), Nelson (1996), and Tomasello (2003). Simply put: The comprehension and use of linguistic symbols, in flexible and communicatively appropriate ways, depend on infants' understanding of others as intentional agents with whom one can share experience. And so to the degree that infants' gestural communication resembles their linguistic communication, similar underlying processes may be inferredproviding still further support for the cognitively "rich" view of infant pointing involving both intentional/mental states and shared intentionality.

Most fundamentally, one of the best-established facts in the study of early language acquisition is the crucial role of joint attentional frames. Bruner (see 1983 for a review) argued and provided evidence that children's comprehension and learning of language is scaffolded by "joint attentional formats" in which both the child and the adult have a common understanding of some delimited domain of experience. Thus, how mothers use language within joint attentional frames relates quantitatively to their children's early language development, whereas mothers' use of language outside of such frames has no such relation - and these relations are evident at around the first birthday, shortly after children's 
earliest pointing (Carpenter et al., 1998; Tomasello \& Farrar, 1986; see Tomasello, 1988, 2003, for reviews of related results). The explanation for these findings is simply that to comprehend an act of linguistic reference the communicator and recipient must connect the linguistic act to some form of common groundjust as in the case of pointing. Thus, hearing a novel word "Gavagai," the infant must find some way to establish joint attention with the speaker on a referent - by connecting to common ground - in the very same way that they do this in comprehending a pointing gesture (see, e.g., Tomasello, 2001, for studies with infants as young as 18 months, and Mundy \& Sigman, 2006, on joint attention and language development in children with autism).

In most cases pointing presupposes the joint attentional common ground as "topic" (old or shared information), and the pointing act is actually a predication, or focus, informing the recipient of something new, worthy of her attention. In other cases, pointing serves to establish a new topic, about which further things may then be communicated. These are two of the main functions served by whole utterances in linguistic communication, what Lambrecht (1994) calls predicate focus and sentence focus constructions. Moreover, when infants first begin talking, many of their earliest utterances are combinations of gestures (mostly pointing) with words, dividing up in various ways the topic and focus functions (Iverson \& Goldin-Meadow, 2005; Ozcaliskan \& GoldinMeadow, 2005; Tomasello, 1988). Language of course goes well beyond pointing and other deictic gestures in the ease with which linguistic symbols may be grammaticalized into constructions with complex topic-focus configurations, but the key point in the current context is simply that the topic-focus building blocks are basically the same in the two cases.

The cooperative motives for communicating linguistically and via pointing are basically the same as well: informing, requesting, and sharing attitudes. (Early in development most children also learn to ask questions, but in most analyses questions are considered requests for information.) Indeed, of Searle's (1999) five speech act functions in adult language, these three are the earliest to emerge ontogenetically. The two others, that children will learn later as they become more cognitively and linguistically sophisticated, are promising (commisives) and declarations such as "I now pronounce you man and wife." Whether someone can promise via pointing or any other nonlinguistic gesture is currently a matter of some debate, as is the possibility of their performing certain kinds of declarations without language. Nevertheless, the basic point is that the most fundamental motives for linguistic communication - those that emerge first ontogenetically - are the same in both pointing and linguistic communication.

Language is of course very different from pointing structurally, as a language is a historically evolved inventory of symbolic devices for directing others' attention perspectivally in myriad ways, that is, directing attention in one way, rather than other ways that are also possible in the symbolic inventory. Linguistic reference thus goes well beyond what it is possible to indicate explicitly by simply indexically directing someone's attention to a location. Nevertheless, in our many examples in which the meaning of pointing changes with the joint attentional frame-pointing to a bicycle may be intended to indicate either the material or the color, for instance - a certain kind of perspective shifting is involved. It is thus possible that the kind of reference shifting in pointing-accomplished by making contact in different ways with communicator-recipient common ground-paves the way for perspectival linguistic symbols both phylogentically and ontogenetically. Additionally, although reference to entities displaced in space and/or time has traditionally been held to be the exclusive provence of language - and there is no doubt that language does this by far most productively - within the appropriate joint attentional frame, even 1-year-old infants may point to indicate something about nonpresent entities.

Our argument is thus that the most fundamental aspects of language that make it such a uniquely powerful form of human cognition and communication-joint attention, reference via perspectives, reference to absent entities, cooperative motives to help and to share, and other embodiments of shared intentionality - are already present in the humble act of infant pointing.

\section{Conclusion}

Pointing things out for other people seems like an exceedingly simple act. But it turns out that this is a uniquely human form of communication under natural circumstances, and it rests on a very complex and mostly hidden social-cognitive, social-motivational infrastructure that, apparently, nonhuman species simply do not possess in anything like the human form. The social-cognitive part of the infrastructure comprises mainly the joint attentional frame, which rests on the ability to know things mutually with others, and the communicative intention that derives from skills of joint attention as it is essentially the 
intention that we know together that I want something from you. The social-motivational part of the infrastructure comprises the cooperative motives of helping (by informing) and sharing (emotions and attitudes) in a communicative context - and indeed these cooperative motives are not just expressed by communicators and understood by recipients; they are mutually assumed (Grice, 1975).

The evidence we have presented here demonstrates - in a variety of different ways - that when pointing first emerges in human infants at around the first birthday, before the emergence of language, it already possesses these foundational components of mature pointing. Infants depend in both their comprehension and production of pointing on a joint attentional frame (common ground) with their communicative partners in order to identify those aspects of things referred to, and even reference to absent entities. They do this on a mental level involving an understanding of the intentions, attention, and knowledge of their partner. And they do this for the fundamentally cooperative motives of helping and sharing information and attitudes with others - and indeed even 1-year-old infants seem already to assume mutually that others are trustworthy and helpful in general, as they readily make relevance inferences when people inform them of things cooperatively. It might also be possible to defend a leaner theory: that infants are instead doing something like using adults as social tools to achieve behavioral goals without understanding the process of communication as a "meeting of minds." But if that were the case, it would be very difficult to explain infants' behavior in the variety of experiments we have reported here, from comprehending points based on shared experience, to pointing to absent referents, to pointing for adults in order to share attitudes, to pointing for adults in order to inform them of something they wish to know.

The evolution of humans' uniquely cooperative form of communication is still something of a mystery, and it is unclear as to the degree to which human forms of cooperation and culture, in general, enable versus depend on, cooperative communication. On the one hand, the cooperative structure of human communication may be just one more manifestation of humans' tendency to do things together, with joint goals, intentions, and attention (shared intentionality). On the other hand, it may be that other forms of multiparty activity may be transformed by cooperative communication. Thus, one can imagine small groups of humans doing such things as hunting together or gathering nuts together without any significant communication among them about this group activity. This would make the forming of shared goals and the coordination of joint plans and intentions difficult, if not impossible. It may thus be, then, that cooperative communication was the original adaptation, and all other forms of cooperative activity involving shared intentionality depend upon it.

In any case, based on our review of recent research on infant pointing, our claim is that in human ontogeny today infants from 12 to 14 months of age, before language acquisition has begun in earnest, already participate in the species-unique activity of human cooperative communication. This fact provides an existence proof that human-style cooperative communication does not depend on language, and rather suggests that language depends on it. Pointing may thus represent a key transition, both phylogenetically and ontogenetically, from nonlinguistic to linguistic forms of human communication.

\section{References}

Bakeman, R., \& Adamson, L. (1984). Coordinating attention to people and objects in mother-infant and peerinfant interactions. Child Development, 55, 1278-1289.

Baron-Cohen, S. (1989). Perceptual role taking and protodeclarative pointing in autism. British Journal of Developmental Psychology, 7, 113-127.

Bates, E., Camaioni, L., \& Volterra, V. (1975). The acquisition of performatives prior to speech. Merrill-Palmer Quarterly, 21, 205-224.

Behne, T., Carpenter, M., Call, J., \& Tomasello, M. (2005). Unwilling versus unable: Infants' understanding of intentional action. Developmental Psychology, 41, 328-337.

Behne, T., Carpenter, M., \& Tomasello, M. (2005). One-yearolds comprehend the communicative intentions behind gestures in a hiding game. Developmental Science, 8, $492-499$.

Bratman, M. (1992). Shared co-operative activity. Philosophical Review, 101, 327-341.

Brinck, I. (2004). The pragmatics of imperative and declarative pointing. Cognitive Science Quarterly, 3, 4.

Bruner, J. (1975). The ontogenesis of speech acts. Journal of Child Language, 2, 1-19.

Bruner, J. (1983). Child's talk. New York: Norton.

Butterworth, G. (2003). Pointing is the royal road to language for babies. In S. Kita (Ed.), Pointing: Where language, culture, and cognition meet (pp. 9-33). Mahwah, NJ: Erlbaum.

Call, J., \& Tomasello, M. (1994). Production and comprehension of referential pointing by orangutans (Pongo pygmaeus). Journal of Comparative Psychology, 108, 307-317.

Call, J., \& Tomasello, M. (2005). What chimpanzees know about seeing revisited: An explanation of the third kind. In N. Eilan, C. Hoerl, T. McCormack, \& J. Roessler (Eds.), 
Joint attention (pp. 45-64). Oxford, UK: Oxford University Press.

Camaioni, L. (1993). The development of intentional communication: A re-analysis. In J. Nadel \& L. Camaioni (Eds.), New perspectives in early communicative development (pp. 82-96). New York: Routledge.

Carpenter, M., Call, J., \& Tomasello, M. (2005). 12- and 18month-olds copy actions in terms of goals. Developmental Science, 8, F13-F20.

Carpenter, M., Nagell, K., \& Tomasello, M. (1998). Social cognition, joint attention, and communicative competence from 9 to 15 months of age. Monographs of the Society for Research in Child Development, 63(4, Serial No. 255).

Clark, H. (1996). Uses of language. Cambridge, UK: Cambridge University Press.

Clark, H., \& Marshall, C. (1981). Definite reference and mutual knowledge. In A. K. Joshi, B. L. Webber, \& I. A. Sag (Eds.), Elements of discourse understanding (pp. 101-131). Cambridge, UK: Cambridge University Press.

Csibra, G. (2003). Teleological and referential understanding of action in infancy. Philosophical Transactions of the Royal Society, London B, 358, 447-458.

D’Entremont, B., Hains, S. M. J., \& Muir, D. W. (1997). A demonstration of gaze following in 3- to 6-month-olds. Infant Behavior and Development, 20, 560-572.

Gergely, G., Bekkering, H., \& Király, I. (2002). Rational imitation in preverbal infants. Nature, 415, 755.

Gergely, G., Nádasdy, Z., Csibra, G., \& Bíró, S. (1995). Taking the intentional stance at 12 months of age. Cognition, 56, 165-193.

Golinkoff, R. (1993). When is communication a meeting of the minds? Journal of Child Language, 20, 199-208.

Grice, P. (1957). Meaning. The Philosophical Review, 64, $377-388$.

Grice, P. (1975). Logic and conversation. In P. Cole \& J. Morgan (Eds.), Syntax and semantics. Vol. 3: Speech acts (pp. 43-58). New York: Academic Press.

Hannan, T., \& Fogel, A. (1987). A case study assessment of "pointing" in the first three months of life. Perceptual and Motor Skills, 65, 187-194.

Hare, B., \& Tomasello, M. (2004). Chimpanzees are more skillful in competitive than in co-operative cognitive tasks. Animal Behaviour, 68, 571-581.

Hare, B., \& Tomasello, M. (2005). Human-like social skills in dogs? Trends in Cognitive Science, 9, 439-444.

Iverson, J., \& Goldin-Meadow, S. (2005). Gesture paves the way for language development. Psychological Science, 16, 367-373.

Kendon, A. (2004). Gesture: Visible action as utterance. Cambridge, UK: Cambridge University Press.

Kita, S. (Ed.). (2003). Pointing: Where language, culture and cognition meet. Mahwah, NJ: Erlbaum.

Kuhlmeier, V., Wynn, K., \& Bloom, P. (2003). Attribution of dispositional states by 12 -month olds. Psychological Science, $14,402-408$.

Lambrecht, K. (1994). Information structure and sentence form. Cambridge, UK: Cambridge University Press.
Leavens, D. A., \& Hopkins, W. D. (1998). Intentional communication by chimpanzees: A cross-sectional study of the use of referential gestures. Developmental Psychology, 34, 813-822.

Leavens, W., Russell, J., \& Hopkins, W. (2005). Intentionality as measured in the persistence and elaboration of communication by chimpanzees. Child Development, 76, 291-306.

Leung, E. H., \& Rheingold, H. L. (1981). Development of pointing as a social gesture. Developmental Psychology, 17, 215-220.

Liebal, K., Behne, T., Carpenter, M., \& Tomasello, M. (2007). Infants use shared experience to interpret pointing gestures. Manuscript submitted for publication.

Liszkowski, U. (2005). Human twelve-month-olds point co-operatively to share interest with and provide information for a communicative partner. Gesture, 5, 135154.

Liszkowski, U. (2006). Infant pointing at twelve months: Communicative goals, motives, and social-cognitive abilities. In N. Enfield \& S. Levinson (Eds.), The roots of human sociality: Culture, cognition, and interaction (pp. 153 -178). Oxford, UK: Berg.

Liszkowski, U., Carpenter, M., Henning, A., Striano, T., \& Tomasello, M. (2004). Twelve-month-olds point to share attention and interest. Developmental Science, 7, 297-307.

Liszkowski, U., Carpenter, M., Striano, T., \& Tomasello, M. (2006). Twelve- and 18-month-olds point to provide information for others. Journal of Cognition and Development, 7, 173-187.

Liszkowski, U., Carpenter, M., \& Tomasello, M. (2007a). Pointing out new news, old news, and absent referents at 12 months of age. Developmental Science, 10, F1-F7.

Liszkowski, U., Carpenter, M., \& Tomasello, M. (2007b). Reference and attitude in infant pointing. Journal of Child Language, 34, 1-20.

Liszkowski, U., Carpenter, M., \& Tomasello, M. (2007c). Twelve-month-olds provide information to help an adult find what she is looking for. Manuscript submitted for publication.

Masataka, N. (2003). From index-finger extension to indexfinger pointing: Ontogenesis of pointing in preverbal infants. In S. Kita (Ed.), Pointing: Where language, culture, and cognition meet (pp. 69-84). Mahwah, NJ: Lawrence Erlbaum.

McNeil, D. (1992). Hand and mind. What gestures reveal about thought. Chicago: University of Chicago Press.

Menzel, C. (1999). Unprompted recall and reporting of hidden objects by a chimpanzee after extended delays. Journal of Comparative Psychology, 113, 426-434.

Miklosi, A., \& Soporoni, K. (2006). A comparative analysis of animals' understanding of the human pointing gesture. Animal Cognition, 9, 81-93.

Moll, H., Koring, C., Carpenter, M., \& Tomasello, M. (2006). Infants determine others' focus of attention by pragmatics and exclusion. Journal of Cognition and Development, 7, 411-430. 
Moll, H., \& Tomasello, M. (2004). 12- and 18-month-olds follow gaze to hidden locations. Developmental Science, 7 , F1 - F9.

Moll, H., \& Tomasello, M. (in press). How 14- and 18month-olds know what others have experienced. Developmental Psychology.

Moore, C. (1996). Theories of mind in infancy. British Journal of Developmental Psychology, 14, 19-40.

Moore, C., \& Corkum, V. (1994). Social understanding at the end of the first year of life. Developmental Review, 14, $349-372$.

Moore, C., \& D'Entremont, B. (2001). Developmental changes in pointing as a function of parent's attentional focus. Journal of Cognition and Development, 2, $109-129$.

Mundy, P., \& Sigman, M. (1989). The theoretical implications of joint attention deficits in autism. Development and Psychopathology, 1, 173-183.

Mundy, P., \& Sigman, M. (2006). Joint attention, social competence and developmental psychopathology. In D. Cicchetti \& D. Cohen (Eds.), Developmental psychopathology (Vol. 1, 2nd ed.). Theory and methods (pp. 79-108). Hoboken, NJ: Wiley.

Nelson, K. (1996). Language in cognitive development. New York: Cambridge University Press.

Onishi, K. H., \& Baillargeon, R. (2005). Do 15-month-old infants understand false beliefs? Science, 308, $255-258$.

Ozcaliskan, S., \& Goldin-Meadow, S. (2005). Gesture is at the cutting edge of language development. Cognition, 96, B101 - B113.

Piaget, J. (1952). The origins of intelligence in children. New York: Norton.

Saylor, M. (2004). 12- and 16-month-old infants recognize properties of mentioned absent things. Developmental Science, 7, 599-611.

Schwier, C., van Maanen, C., Carpenter, M., \& Tomasello, M. (2006). Rational imitation in 12-month-old infants. Infancy.

Searle, J. (1995). The construction of social reality. New York: Free Press.

Searle, J. R. (1999). Mind, language and society. Philosophy in the real world. New York: Basic Books.

Shatz, M. (1983). Communication. In P. H. Mussen (Series Ed.), J. Flavell \& E. Markman (Vol. Eds.), Handbook of child psychology: Vol. 3. Cognitive development (4th ed., pp. 841 -889). New York: Wiley.

Shatz, M., \& O'Reilly, A. (1990). Conversation or communicative skill? A re-assessment of two-year-olds' be- havior in miscommunication episodes. Journal of Child Language, 17, $131-146$.

Shwe, H., \& Markman, E. (1997). Young children's appreciation of the mental impact of their communicative signals. Developmental Psychology, 33, 630-636.

Sperber, D., \& Wilson, D. (1986). Relevance: Communication and cognition. Cambridge, MA: Harvard University Press.

Tomasello, M. (1988). The role of joint attentional process in early language development. Language Sciences, 10, $69-88$.

Tomasello, M. (2001). Perceiving intentions and learning words in the second year of life. In M. Bowerman \& S. Levinson (Eds.), Language acquisition and conceptual development (pp. 132-158). Cambridge, UK: Cambridge University Press.

Tomasello, M. (2003). Constructing a language: A usage-based theory of language acquisition. Cambridge, MA: Harvard University Press.

Tomasello, M. (2006). Why don't apes point? In N. J. Enfield \& S. C. Levinson (Eds.), Roots of human sociality: Culture, cognition, and interaction (pp. 506-524). Oxford, UK: Berg.

Tomasello, M., \& Camaioni, L. (1997). A comparison of the gestural communication of apes and human infants. Human Development, 40, 7-24.

Tomasello, M., Carpenter, M., Call, J., Behne, T., \& Moll, H. (2005). Understanding and sharing intentions: The origins of cultural cognition. Behavioral and Brain Sciences, $28,675-735$.

Tomasello, M., \& Farrar, J. (1986). Joint attention and early language. Child Development, 57, 1454-1463.

Tomasello, M., \& Haberl, K. (2003). Understanding attention: 12- and 18-month-olds know what's new for other persons. Developmental Psychology, 39, 906-912.

Vea, J., \& Sabater-Pi, J. (1998). Spontaneous pointing behaviour in the wild pygmy chimpanzee (Pan paniscus). Folia Primatologica, 69, 289-290.

Warneken, F., \& Tomasello, M. (2006). Altruistic helping in human infants and young chimpanzees. Science, 31, $1301-1303$.

Warneken, F., \& Tomasello, M. (in press). Helping and cooperation at 14 months of age. Infancy.

Werner, H., \& Kaplan, B. (1963). Symbol formation: An organismic developmental approach to language and the expression of thought. New York: John Wiley.

Wittgenstein, L. (1955). Philosophical investigations. Oxford, UK: Basil Blackwell. 